\title{
FORTY-FIVE YEARS OF RESEARCH AND TEACHING
}

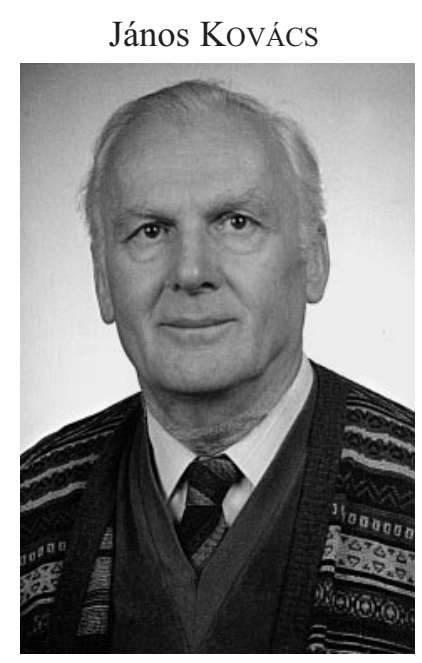

I am greatly honoured by the invitation of the Editors of Acta Biologica Hungarica to write about my scientific career, however, I do it with some reluctance because only time can decide what is really valuable and important in one's views and results for the development of science.

The University of Budapest named after Eötvös Loránd, the famous Hungarian physicist has been the scene of my work that was deeply influenced by the spirit and traditions of this more than 360 years old institution. In contrast to its more lucky counterparts in Western Europe which could develop safely during their history, this university was enforced to grow and exist centuries long in the harsh political and economical environment of a small Central European country often restricted in its independence. We learned to live and survive according to the famous slogan: "fluctuat nec mergitur".

My career in science began in 1956 when after completing my university studies in biology I had the opportunity to work in the laboratory of Prof. G. Mödlinger, the Head of the Dept. of Comparative Anatomy. Prof. Mödlinger was a well-known Hungarian endocrinologist engaged in the study of circadian rhythm of the thyroid gland. He offered me to study the circadian activity of testicular Leydig cells and I soon was able to show diurnal fluctuations in the volume of their nuclei, which was in good correlation with blood androgen levels. The volume of nuclei was determined by morphometric evaluation and morphometry was frequently used in my later studies as well. This was a heroic era of rapid development of electron microscopy and cell fractionation (the tremendous success achieved in cell biology by the application of these techniques was later acknowledged by Nobel prizes awarded to Palade, Claude and DeDuve). These, together with the arsenal of newly developed 
morphological, histochemical, physiological and biochemical methods opened up new possibilities for the complex characterization of cell functions. I soon realized that without their application my work cannot be successful. Unfortunately, however, no financial support could be obtained at that time to buy and install the expensive instruments and therefore, to join this trend seemed hopeless for me. The year 1959 was a turning point in my scientific career. I got a chance to continue my studies as an aspirant (PhD student) in cell biology at the University of Leningrad (now Saint-Petersburg). I was deeply impressed by the cultural atmosphere and scientific traditions of this beautiful city. I noticed with a great surprise and satisfaction that "lisenkoism" the dominant official biological dogma of those days in the Sovietunion had no influence on the scientific community of this university. They were welltrained and excellently informed about the latest achievements in their field and rejected the pseudoscientific stupidities. Leningrad was the housing city of the most famous cytological school of the Sovietunion. The school was founded by Prof. D. Nassonow, a leading expert in the research of Golgi complex, at the early 1930s but its roots could be traced back to the seminal works of I. Metscnikoff on phagocytosis. First, I began my work at the Dept. of Genetics and later on I continued at the Dept. of Cytology, headed by my supervisor, Prof P. Makarov. Makarov and other members of Nassonow's group studied the phenomenon paranecrosis. This was the term coined to describe the reversible morphological and biochemical changes observed in mildly injured cells. Vital staining of cells by basic vital dyes was the most popular and convenient method to study paranecrosis. During this process some basic vital dyes, e.g. neutral red accumulate in granular form in intact cells. In contrast, in injured cells diffuse staining of the whole cytoplasm is observed as a rule and the amount of bound dye greatly exceeds that of measured in the intact ones. It was supposed that these changes were caused by the unfolding and denaturation of the cell proteins. The idea that the reaction of a cell may be explained by the flexibility and reversible conformational changes of its proteins was entirely new at that time. Unfortunately, in conditions of total international isolation and without opportunity to publish their results in international journals, the works of Nassonow's group remained almost unnoticed in the scientific communities of western countries.

In Makarov's lab my task was to characterize the events of paranecrosis by histochemical methods. It was soon revealed that the granules stained by vital dyes in intact cells are lysosomes and I could describe the changes in localization and activities of a series of enzymes including acid phosphatase and others in injuried cells. This was the era of discovery of lysosomes by DeDuve's group and the rapid progress in this field inspired me a lot. Since that time all of my experimental works has been connected tightly or loosely to the research of these organelles.

After obtaining the candidate's degree in Leningrad in 1962, I returned to the Dept. of Comparative Anatomy of Eötvös Loránd University and was offered the chance to organize a laboratory of cell biology. Later on, in 1969 after the retirement of Prof. Mödlinger, I was appointed as Head of the department. In the course of several years we organized laboratory of electron microscopy, succeeded to get support for obtaining instruments for cell fractionation and, most importantly, young colleges 
and students joined us (many of them are now leaders of their own research group) and as a result a research group in cell biology was established for the first time in the history of our university.

Inspection of cells stained by neutral red and other vital dyes revealed that besides typical lysosomes they always contained many round bodies bordered by a single membrane or membrane pairs and filled up with fragments of cell components (mainly with parts of endoplasmic reticulum). The first data on autophagy were published in the late 1960s and this was immediately followed by a rapid and steadily rise of publications on this subject. Comparing our micrographs with those freshly published in the literature it became evident that the bodies formed in the vitally stained cells are true autophagic vacuoles. This was a decisive moment and since this time most of my scientific activities has been aimed at elucidating the mechanism and role of autophagy in the cells.

Autophagy is a multistep process, which begins with the segregation of portions of cytoplasm into autophagosomes by a double membrane (often called the isolating cistern). Autophagosomes mature into autolysosomes by fusion with members of the endosomal/lysosomal system and their content becomes degraded. The origin and mechanism of formation of isolating membrane is still uncertain in spite of continuing efforts during the last 40 years to solve this problem. Autophagy is an important, in some circumstances dominant way of the degradation of intracellular proteins. Our first efforts were aimed at the elucidation of the sequence of steps and the characterization of the kinetics of the autophagic process. We discovered that at least in murine tissues in vivo the first step (segregation) can be rapidly and totally suppressed by the well-known inhibitor of protein synthesis cycloheximide and observed an exponential decay in the volume fraction of autophagic vacuoles in cycloheximide-treated cells and also showed that the half-life of autophagic vacuoles is about 6-8 min. We studied the effect on autophagy of the well-known antimicrotubular drug vinblastine and showed that this agent caused a tremendous increase of the volume fraction of autophagic vacuoles. Vinblastine acts on the autophagic process at two levels: it inhibits the fusion between autophagosomes and lysosomes causing thereby the expansion of the former and, on the other hand, it stimulates the process of segregation, at least in murine pancreatic cells. Later on, a more detailed analysis of the effects of various agents acting on autophagy was accomplished by A. L. Kovács on isolated liver cells in the lab of Prof. Seglen (Oslo) who developed, and applied this method for the study of autophagy. Presently, the method has been futher developed and adapted for isolation of pancreatic acini by A. L. Kovács and A. Telbisz, which permitted them for the first time to follow the autophagic process with concommitant measurement of the rate of intracellular proteolysis in this type of tissue. Their data show that significant differences may exist in the regulation of autophagy between liver and pancreatic cells.

In another series of experiments, we tried to elucidate the origin and mode of formation of isolating membranes. In spite of many efforts the mechanism of this key process of autophagy is yet unresolved. Nevertheless, we obtained some important data on this issue. Earlier, the membranes of endoplasmic reticulum were considered 
by many authors as direct source of isolating membranes. In our lab this question was investigated by G. Réz and his group. They observed detachment of the ribosomes from the membranes of RER under conditions of stimulated autophagy and came to the conclusion that degranulation reflecting labilization or change of RER is a prerequisite for the autophagic reaction. Later on, G. Réz, working in the laboratory of Prof. J. Meldolesi, applied the freeze-fracture method for the study of isolating membranes of autophagic vacuoles and showed for the first time that they are almost free of intramembrane particles and represent an unique class of intracellular membranes distinct from any other preformed membranes. An important conclusion of this work was that direct transformation of ER membranes into isolating membrane seems to be improbable. On the other hand, these experiments indicated, that the questions of autophagy cannot be solved by only applying conventional electronmicroscopic methods. Therefore, methods were developed in our lab for the isolation and purification of autophagic vacuoles and we were the first who could obtain pure fraction of autophagosomes.

As a rule, autophagy proceeds with low intensity in most types of intact cells. Therefore, agents stimulating the process are frequently applied in the experiments. However, cell-systems exist in which intense autophagy is induced spontaneously by intrinsic physiological factors in well-defined stage of development. Larval tissues of holometabolous insects are prominent examples of these systems, in which tremendous autophagic wave can be observed during metamorphosis under hormonal influence. We found promising to work in this field and begin to carry out experiments with insect larval tissues. This was a good decision with far reaching consequences. Beginning from the early 1970s years M. Sass and L. Kömúves published a series of papers on the autophagic process in the larval fat body and midgut cells of Mamestra and Pieris species. Later on, M. Sass continued his work in the laboratory of Prof. M. Locke (Univ. of Western Ontario) and after returning to Budapest, he organized a large lab at our department. Now here, they successfully use insect cells for study of a broad spectrum of key problems of cell biology such as autophagy, and sorting and targeting of secretory proteins.

Autophagy is a common phenomenon in cells exposed to injurious, pathological conditions. This fact prompted us to initiate studies on the role of proteolytic processing of proteins playing role in the development of neurodegenerative disorders. Presently, L. László, who became familiar with this subject in labs of R. Mayer and S. Prusiner, carries out immunocytochemical studies on cells infected with prion proteins, an issue which became extremely important in the last few years.

During the last two years we witnessed a period of breakthrough in the research of autophagy. Several genes governing autophagy were successfully isolated for the first time, among which kinases and ubiquitin-like conjugating enzymes were found. New data were obtained on the structure of isolating membranes which strengthen our previous conclusion on the unique nature of these membranes. These new studies were carried out on $S$. cerevisiae and related species. In our department, now headed by Prof. M. Sass two groups investigate the genetical background of autophagy on more complex multicellular organisms, Drosophila and C. elegans. 
Altough I am more a spectator than an active participant of these investigation, it is a pleasure to notice that my friends and co-workers who started their scientific career in my lab are now in the frontiers of autophagy research. This strengthens my conviction that the decision made some thirty years ago to focus our efforts on study of autophagy was right. We made some interesting discoveries in this field, which - more importantly - offered opportunity for the young members of our group to test their creativity and productivity and opened the doors of leading foreign laboratories for further research. Presently, most of them are leaders of separate teams investigating a wide array of problems which are, however, tightly or loosely connected to the phenomenon of autophagy.

In the last years my attention was mostly directed to the investigation of microtubules. This was partly prompted by the fact, that microtubuli play a prominent role in the regulation of autophagy. On the other hand, ample data suggest that many cytosolic proteins are reversibly associated to the elements of the cytoskeleton among which glycolytic enzymes are found, as was shown by J. Ovadi in the Institute of Enzymology of HAS. Indeed, in a series of joint experiments we provided electron microscopical evidence for the binding of phosphofructokinase, aldolase and pyruvate kinase to the surface of microtubules and showed that this association may influence both the stability of microtubules and the activity of the enzyme. These data strengthen the view that besides its cytoskeletal function, the microtubular network is an important regulator of the activity and localisation of many enzymes. It is extremely interesting that two of the proteins capable to bind to the microtubuli, phosphofructokinase and the LC3 subunit of MAP1 proved to be regulators of the formation of autophagic vacuoles. It was a great satisfaction to discover hidden connection between phenomena which seemed distant and unrelated before.

I was always strongly motivated to explain and teach cell biology and in our department I found ample possibilities to follow this attraction. Even now, with 40 years teaching experience, I enjoy teaching and discussions with students as much as in those early days when I began my work in the university. At that time cell biology was taught within the frame of courses on anatomy and histology and students got acquainted mainly with data on morphology of the cells as seen in the light microscope. Simultaneously with starting of cytological research work in the department, I began to organize separate basic courses on cell biology. Later on, textbooks and an atlas of electronmicroscopical morphology of the cell were prepared which in revised and extended form is used yet at the department. During several years a teaching staff of young colleges well trained in cell biology was formed. Now, besides basic course students are offered a wide variety of advanced courses on recent progress in cell biology together with practical courses on methods of research work in cytology. Finally, we organized a PhD school on molecular cell biology at the department in which more then 30 postgraduate students prepared or are preparing their thesis work during the last six years.

Finally, I wish to say a word about my activities in the organization of research and education in the Institute of Biology that is the biggest one of our faculty. I never found much enjoyment in this type of work but considered the management of affairs 
useful and necessary for keeping our community competitive and adaptive to the changes in science and in social/economical environment of the country as well. I was elected director of the Institute of Biology in two critical periods of the contemporary history of our faculty. Firstly, in the early 1970s years when we were forced to find answers to new challenges in science and teaching caused by the rapid development of bioscience. The Institute as a whole was reorganized, two new departments (Dept. of Immunology, Dept. of Ethology) were established, and new heads were appointed to more then half of the already existing ones. The changes were really profound and were not accepted without difficulties but were justified by later events. Now, more than one hundred scientists working in 12 departments take a part in teaching and research at our institute which is the biggest biological unit among those of Hungarian universities. The second critical period fell on the first part of 1990s. This was the period of transition of the political system of this country into democracy accompanied by profound changes in the economy and the life of the society. As a consequence, the environment surrounding the university was also deeply changed. The university was given true autonomy, the number of students greatly increased, however, the funds supporting teaching and research became inadequate and in 1995-1996, due to the economical crisis of the country, we were compelled to dismiss about $20 \%$ of our teaching staff. During this hard period we tried to maintain the traditionally high level of teaching and research and to preserve our greatest treasure: the core of the community of well-trained scientists. It is not my task to judge how successful this policy has been, especially because the process of transformation is not yet completed either in our university or in the whole system of higher education of the country. Nevertheless, in spite of all controversies and due to the effort of devoted people the Institute of Biology exists and fulfills all of its tasks.

In conclusion, I hope that I have been able build a few bricks into the building of science and contribute to sustain the capability of this old university for continuous growth and renewal. I am satisfied with the fact that the department I headed through many years is now strong and flourishing and I hope I could call the attention of my friends and coworkers to some new and interesting problems of cell biology and my ideas helped them to achieve success in science. I am greatly honored to have had the possibility to work and continue working in this country in this institute and in this age. 